\title{
Una nueva mutación asociada con el síndrome de Pierson
} A new mutation associated with Pierson syndrome

\author{
Dr. Ferit Kulali, Dra. Sebnem Calkavur ${ }^{a}$, Dra. Cemaliye Basaran ${ }^{b}$, Dr. Erkin Serdaroglu ${ }^{b}$, Dra. Melis Kose ${ }^{c} y$ \\ Dra. Merve Saka Guvenc ${ }^{d}$
}

\section{RESUMEN}

El síndrome de Pierson se caracteriza por la presencia de síndrome nefrótico congénito y microcoria bilateral. Genéticamente, este trastorno está ocasionado por mutaciones en el gen $L A M B 2$, que codifica la cadena $\beta 2$ de la laminina. Hasta la fecha, en la bibliografía se informaron 98 casos y 50 mutaciones diferentes. No existen terapias específicas para el síndrome de Pierson, y el tratamiento es complementario. El pronóstico es malo por la disfunción renal progresiva y las complicaciones de la insuficiencia renal. En este artículo, se informa sobre una mutación homocigota novedosa (c.1890G>C [p.Q630H]) en el gen $L A M B 2$ en una paciente con síndrome de Pierson que tenía un fenotipo atípico, como epidermólisis ampollosa.

Palabras clave: síndrome de Pierson, síndrome nefrótico, epidermólisis ampollosa, secuenciación del exoma completo, lactante.

http: / / dx.doi.org/10.5546/ aap.2020.e288

Texto completo en inglés:

http: / / dx.doi.org/10.5546/aap.2020.eng.e288

Cómo citar: Kulali F, CalkavurS, Basaran C, Serdaroglu E, et al. Una nueva mutación asociada con el síndrome de Pierson. Arch Argent Pediatr 2020;118(3):e288-e291.

a. División de Neonatología, Hospital de Formación e Investigación de Enfermedades y Cirugías Pediátricas Dr. Behçet Uz, Esmirna, Turquía.

b. División de Nefrología Pediátrica, Hospital de Formación e Investigación de Enfermedades y Cirugías Pediátricas Dr. Behçet Uz, Esmirna, Turquía.

c. División de Metabolismo Pediátrico, Hospital de Formación e Investigación de Enfermedades y Cirugías Pediátricas Dr. Behçet Uz, Esmirna, Turquía.

d. Departamento de Genética Médica, Hospital de Formación e Investigación de Tepecik, Esmirna, Turquía.

Correspondencia:

Dr. Ferit Kulali: fkulali@hotmail.com

Financiamiento: Ninguno.

Conflicto de intereses: Ninguno que declarar.

Recibido: 25-3-2019

Aceptado: 28-10-2019

\section{INTRODUCCIÓN}

El síndrome nefrótico congénito (SNC) está compuesto de trastornos clínica y genéticamente heterogéneos. ${ }^{1}$ Desde una perspectiva etiológica, se divide en SNC primario y secundario. Mientras que los trastornos genéticos en la microestructura glomerular causan SNC primario, las infecciones perinatales producen SNC secundario. ${ }^{2}$ Algunos de los genes implicados en la etiología del SNC primario son NPHS1, NPHS2, WT1, LAMB2 y PLCE1. ${ }^{3}$ Las lamininas son el componente principal de la membrana basal y tienen un papel importante en la adhesión, proliferación, diferenciación y migración de las células. La isoforma $\beta 2$ (laminina $\beta 2$ ) se encuentra, especialmente, en la membrana basal glomerular, las estructuras oculares y las sinapsis neuromusculares. ${ }^{4}$ Las mutaciones en el gen $L A M B 2$, que codifica la laminina $\beta 2$, causan el síndrome de Pierson. En 1963, Pierson y cols. ${ }^{4}$ describieron este síndrome por primera vez, que se caracteriza por SNC y microcoria, que es la manifestación clínica más prominente de un trastorno del desarrollo ocular complejo. Hasta la fecha, se informaron 98 casos y 50 mutaciones diferentes. ${ }^{5}$

En muchos de los casos descriptos previamente, se informaron insuficiencia renal crónica temprana, trastornos graves del neurodesarrollo, microcefalia y ceguera. ${ }^{4}$ No existen terapias específicas para el síndrome de Pierson, y el tratamiento es complementario. ${ }^{6}$

El pronóstico suele ser malo dadas la disfunción renal progresiva y las complicaciones de la insuficiencia renal. ${ }^{7}$

En este artículo, se informa sobre una mutación homocigota novedosa en el gen LAMB2 en una paciente con síndrome de Pierson que tenía un fenotipo atípico, como epidermólisis ampollosa.

\section{Caso:}

Se asistió a una lactante de 51 días de vida con tumefacción en los ojos y las piernas de 10 días de evolución. Era la primera hija de padres consanguíneos sanos, sin antecedentes 
familiares de trastornos genéticos conocidos. Nació por cesárea a las 34 semanas de gestación con un peso al nacer de $1835 \mathrm{~g}$ (percentilo 10-25). No se registró el peso placentario. Sin embargo, durante el embarazo, a la madre se la controló atentamente debido a un retraso del crecimiento intrauterino y oligohidramnios.

$\mathrm{Al}$ momento del ingreso, la paciente pesaba $2980 \mathrm{~g}$ (percentilo 10) y medía $45 \mathrm{~cm}$ (por debajo del percentilo 3). Tenía edema en diversas partes del cuerpo, como la cara, las manos, las piernas, la espalda y los genitales. Su presión arterial era de 113/53 (67) mmHg. La evidencia de hipoproteinemia $(2,2 \mathrm{~g} / \mathrm{dl})$, hipoalbuminemia $(0,9 \mathrm{~g} / \mathrm{dl})$ y proteinuria ( $3+$ proteínas en orina por tira reactiva) confirmó el diagnóstico de SNC. La concentración de albúmina y el cociente proteína/ creatinina en la muestra puntual de orina fueron altos ( $>500 \mu \mathrm{g} / \mathrm{ml}$ y 186,4 , respectivamente). La concentración sérica de creatinina fue de $2,4 \mathrm{mg} /$ dl y la de sodio, $117 \mathrm{mmol} / 1$. Las pruebas de detección metabólicas y las pruebas serológicas de toxoplasmosis, sífilis, rubéola, citomegalovirus, virus del herpes tipo 1 y tipo 2, hepatitis B y C
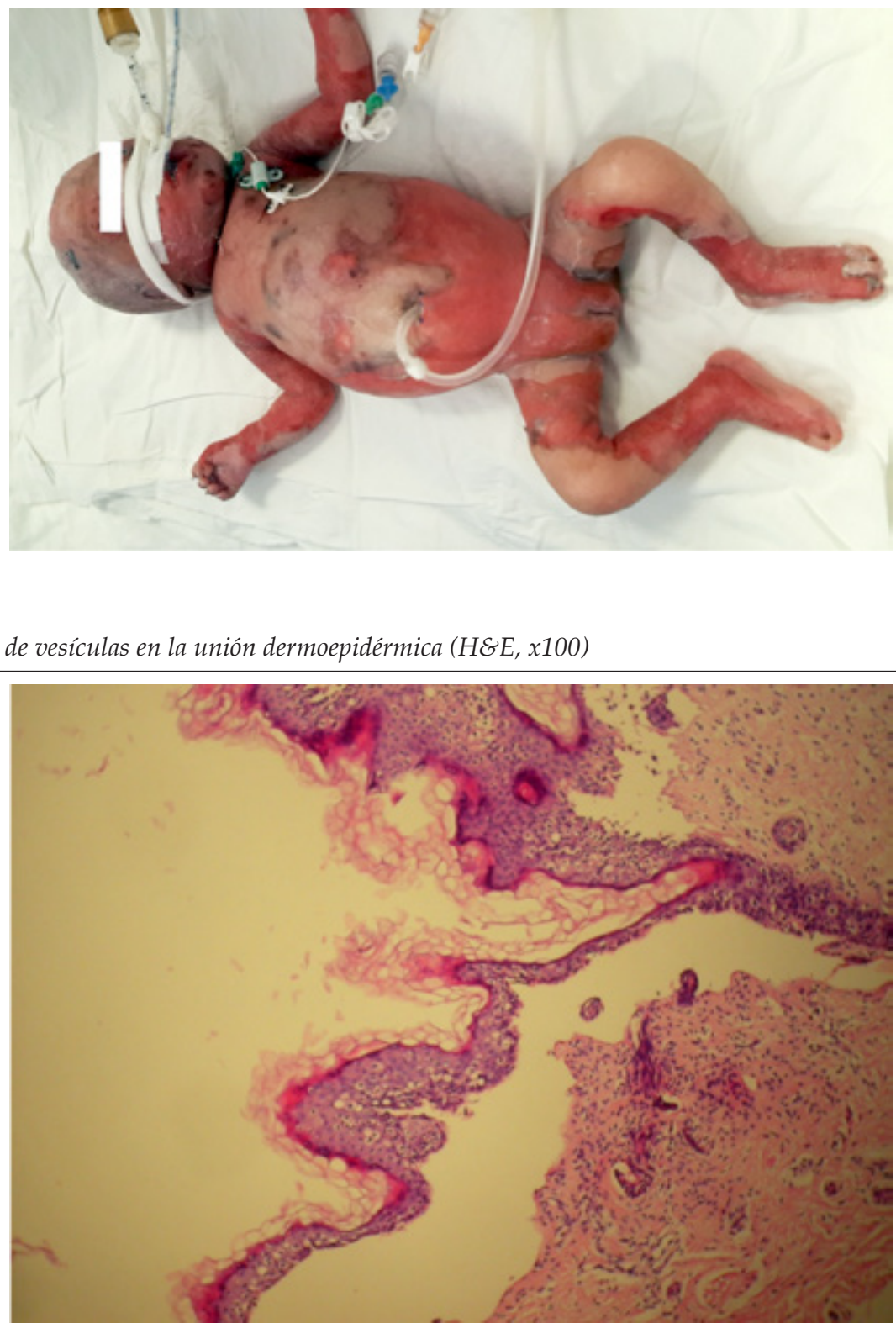
y VIH fueron negativas. En la ecografía renal, se observó que los riñones tenían un tamaño normal, con una ecogenicidad aumentada del parénquima y pérdida de la diferenciación corticomedular. Debido a una midriasis incompleta tras la aplicación de atropina, no fue posible realizar una evaluación ocular detallada. Se detectó microcoria y disgenesia del segmento anterior.

A los 60 días, las lesiones ampollosas en los párpados se extendieron rápidamente al tronco y a las extremidades (Figura 1). La biopsia de las lesiones ampollosas fue compatible con epidermólisis ampollosa de la unión dermoepidérmica (Figura 2). Los padres se negaron a someter a la bebé a una biopsia renal. Inicialmente, se trató a la paciente con infusión de albúmina y furosemida. Sin embargo, a pesar del tratamiento médico adecuado, presentaba anuria y tenía disfunción renal en curso y acidosis metabólica resistente a la administración de bicarbonato. A los 90 días, se la sometió a diálisis peritoneal, tras lo cual, el edema desapareció y las concentraciones de sodio y creatinina en sangre y la presión arterial volvieron a valores normales. Para el tratamiento de las lesiones cutáneas, se aplicó un apósito lípido-coloide $\left(\right.$ Urgotul $\left.^{\circledR}\right)$ y furacin tópico. La necesidad de la infusión de albúmina se redujo gradualmente en el plazo de seis semanas. La paciente falleció a los 129 días de tratamiento debido a una infección.

Figura 3. Resultados del análisis genético del gen LAMB2 de la paciente y sus padres



Estudios genéticos: a los 54 días de vida, el resultado de un cariotipado previo no mostró aberraciones cromosómicas numéricas ni estructurales. Debido al SNC, se realizó un análisis de la secuencia genética de los genes NPHS1, NPHS2 y WT1, que fue normal. A los 74 días de vida, se hizo una secuenciación del exoma completo debido a la asociación inusual de SNC y epidermólisis ampollosa y se detectó un cambio homocigoto c.1890G $>$ C (p.Q630H) en el exón 14 del gen $L A M B 2$. Luego se confirmó este hallazgo mediante una secuenciación de Sanger a los 77 días de vida. Ambos padres eran heterocigotos para la misma alteración (Figura 3).

Se informó a la familia que el riesgo de tener un nuevo hijo con esta afección era del $25 \%$ con cada embarazo. Se habló sobre el diagnóstico prenatal y las opciones de diagnóstico genético preimplantatorio para embarazos futuros.

\section{DISCUSIÓN}

El síndrome de Pierson es un motivo muy raro de $\mathrm{SNC}^{8}$ y está causado por mutaciones en el gen $L A M B 2$, que codifica las lamininas, componentes clave de la membrana basal. El gen LAMB2 se encuentra en el cromosoma 3p21.31 y es de herencia autosómica recesiva. ${ }^{9}$ Este síndrome fue descripto por primera vez en 1963 en dos hermanas con SNC y microcoria. ${ }^{10}$ Presentaban síndrome nefrótico grave que apareció en el período neonatal, avanzó rápidamente a insuficiencia renal terminal y les provocó la muerte antes de las dos semanas de vida. En el caso aquí descripto, la consanguinidad fue compatible con la herencia autosómica recesiva. Debido a la presencia de proteinuria, hipoalbuminemia y edema, inicialmente se diagnosticó a la paciente con SNC; pero los hallazgos adicionales, como presión arterial elevada y microcoria, no coincidían con el SNC típico. En la ecografía renal, se observaron signos coincidentes con SNC, como pérdida de la diferenciación corticomedular y aumento del tamaño renal y de la ecogenicidad del parénquima. La biopsia de riñón se usa para confirmar el diagnóstico, pero no resulta útil para identificar la etiología preexistente y no siempre permite obtener resultados confiables. ${ }^{2}$ Dado que la laminina es uno de los principales componentes de la membrana basal glomerular (MBG), se prevé que ocurran algunos cambios estructurales en la MBG de los pacientes con síndrome de Pierson. ${ }^{11}$ Los hallazgos más importantes de la biopsia renal son aumento difuso de la matriz mesangial o 
lesiones por glomeruloesclerosis segmentaria y focal, ausencia o disminución marcada de la MBG correspondiente a la cadena $\beta 2$ de la laminina y zonas engrosadas y delgadas irregulares en ambos lados de la lámina densa de la MBG con lamelación. ${ }^{12} \mathrm{El}$ diagnóstico diferencial incluye otras causas de síndrome nefrótico congénito / temprano con MBG anormal, como síndrome de Galloway-Mowat. ${ }^{4,12}$ No fue posible realizar una biopsia renal en nuestro caso porque los padres se negaron. En la secuenciación del exoma completo, se observó que la paciente era homocigota para una mutación novedosa en el gen LAMB2 (c.1890G>C [p.Q630H]). Una vez evaluada en conjunto, se diagnosticó síndrome de Pierson.

Este trastorno afecta varios órganos, en especial, los riñones y los ojos. El compromiso renal abarca un amplio rango de manifestaciones, desde destrucción leve de los podocitos hasta esclerosis mesangial difusa. ${ }^{11}$ Suele manifestarse en el período prenatal o en el período posnatal inmediato. En el caso de esta paciente, el retraso del crecimiento intrauterino, el oligohidramnios y la concentración elevada de creatinina sugieren una manifestación prenatal.

El compromiso ocular es el síntoma más importante de esta enfermedad, y la microcoria es el hallazgo ocular más frecuente. También se han descripto otras anomalías oculares, como anomalías del iris y de la retina, cataratas, forma anormal del cristalino y miopía alta. ${ }^{13} \mathrm{En}$ concordancia con la bibliografía, nuestra paciente presentaba microcoria y disgenesia del segmento anterior.

Si bien muchos pacientes con síndrome de Pierson tienen trastornos del neurodesarrollo graves y debilidad muscular, ${ }^{9}$ nuestra paciente no presentaba dicha evidencia. Cabe destacar que, en la biopsia cutánea, se observó epidermólisis ampollosa de la unión dermoepidérmica. La epidermólisis ampollosa es un trastorno genético causado por mutaciones en los genes que codifican las proteínas estructurales de la piel. ${ }^{14}$ Este trastorno incluye cuatro subgrupos principales: simple, de la unión dermoepidérmica, distrófica y síndrome de Kindler. ${ }^{14}$

La coexistencia de síndrome nefrótico, microcoria y epidermólisis ampollosa de la unión dermoepidérmica en nuestra paciente sugiere una mutación en la laminina, una proteína que es un componente importante de la membrana basal renal, ocular y cutánea.
No existen terapias específicas para el síndrome de Pierson, y el tratamiento es complementario. ${ }^{6}$ Este incluye el manejo para lograr el equilibrio hidroelectrolítico, además de la transfusión de albúmina y la diálisis, según sean necesarias. El trasplante renal es la única opción para los pacientes con síndrome de Pierson con insuficiencia renal terminal. ${ }^{7}$ El pronóstico es malo por la disfunción renal progresiva y las complicaciones de la insuficiencia renal. ${ }^{7}$

A modo de conclusión, también es necesario evaluar a los pacientes con SNC para detectar el compromiso de otros órganos, y las pruebas diagnósticas también deberían incluir el análisis genético.

\section{REFERENCIAS}

1. Wang J, Mao JH. The etiology of congenital nephrotic syndrome: current status and challenges. World J Pediatr. 2016; 12(2):149-58.

2. Pleasant LD, Kiessling SG. Congenital nephrotic syndrome. En: Chishti AS, AlamS, KiesslingSG, eds. Kidney and Urinary Tract Diseases in the Newborn. Heidelberg: Springer; 2014. Págs.275-86.

3. Downie ML, Gallibois C, Parekh RS, Noone DG. Nephrotic syndrome in infants and children: pathophysiology and management. Paediatr Int Child Health. 2017; 37(4):248-58.

4. Kagan M, Cohen AH, Matejas V, Vlangos C, Zenker M. A milder variant of Pierson syndrome. Pediatr Nephrol. 2008; 23(2):323-7.

5. Beaufils C, Farlay D, Machuca-Gayet I, Fassier A, et al. Skeletal impairment in Pierson syndrome: Is there a role for laminin $\beta 2$ in bone physiology? Bone. 2018; 106:187-93.

6. Chew C, Lennon R. Basement Membrane Defects in Genetic Kidney Diseases. Front Pediatr. 2018;6:11.

7. Guler S, Cimen S, Acott P, Whelan K, Molinari M. Kidney transplantation in a child with Pierson syndrome. Pediatr Transplant. 2017; 21(8):e13076.

8. Lionel AP, Joseph LK, Simon A. Pierson syndrome - a rare cause of congenital nephrotic syndrome. Indian J Pediatr. 2014; 81(12):1416-7.

9. Matejas V, Hinkes B, Alkandari F, Al-Gazali L, et al. Mutations in the human laminin $\beta 2$ (LAMB2) gene and the associated phenotypic spectrum. Hum Mutat. 2010; 31(9):992-1002.

10. Pierson M, Cordier J, Hervouuet F, Rauber G. An unusual congenital and familial congenital malformative combination involving the eye and kidney. J Genet Hum. 1963; 12:184-213.

11. Choi HJ, Lee BH, Kang JH, Jeong HJ, et al. Variable phenotype of Pierson syndrome. Pediatr Nephrol. 2008; 23(6):995-1000.

12. Lusco MA, Najafian B, Alpers CE, Fogo AB. AJKD Atlas of Renal Pathology: Pierson Syndrome. Am J Kidney Dis. 2018; 71(4):e3-4.

13. Zemrani B, Cachat F, Bonny O, Giannoni E, et al. A novel LAMB2 gene mutation associated with a severe phenotype in a neonate with Pierson syndrome. Eur J Med Res. 2016; 21:19.

14. Fine JD, Bruckner-Tuderman L, Eady RAJ, Bauer EA, et al. Inherited epidermolysis bullosa:updated recommendations on diagnosis and classification. J Am Acad Dermatol. 2014; 70(6):1103-26. 